\title{
Entlassmanagement - DKG klagt gegen Schiedsspruch
}

Die Deutsche Krankenhaus Gesellschaft (DKG) klagt gegen den vom erweiterten Bundesschiedsamt festgelegten „Rahmenvertrag Entlassmanagement". Die DKG hält das „bürokratische Monster“ in zentralen Teilen für rechtwidrig, da das nun festgelegte System nicht von den Intentionen des Gesetzgebers gedeckt sei.

\section{Bundesschiedsamt legt Rahmenvertrag fest}

Die Krankenhausbehandlung umfasst für GKV-Patienten auch ein sogenanntes Entlassmanagement zur Unterstützung einer sektorübergreifenden Versorgung der Versicherten, also beim Übergang von einer stationären in eine ambulante Behandlung (siehe auch [1, 2]). Die Regelungen zum Entlassmanagement sollen zum 01.07.2017 in Kraft treten.

Neben der gesetzlichen Grundlage in $\S 39$ Abs. 1 a SGB $V$ und den Regelungen in der Arzneimittel-Richtlinie fehlte als letzter Schritt zur Umsetzung noch der Rahmenvertrag auf Bundesebene. Dort werden

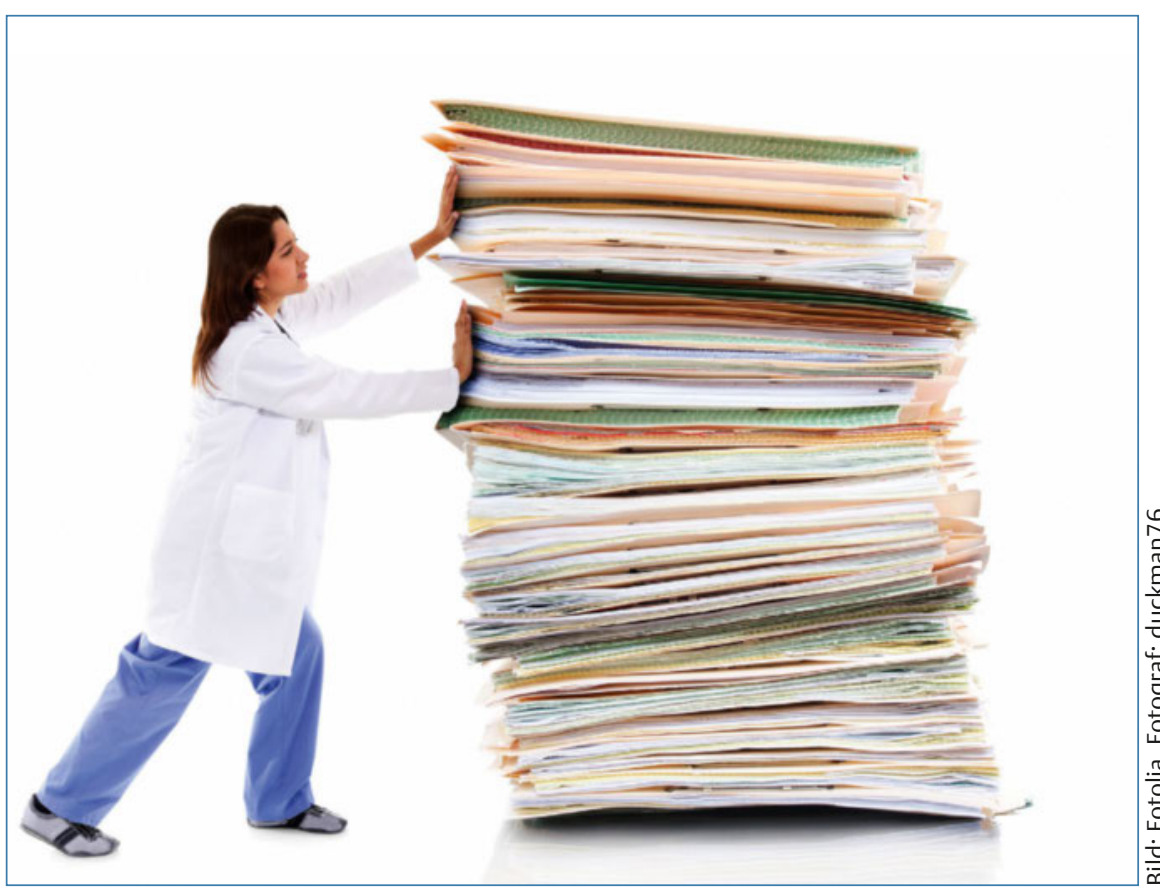

weitere Details für die Umsetzung in die Praxis geregelt. Da sich die Vertragsparteien (Spitzenverband Bund der Krankenkassen (auch als Spitzenverband Bund der Pflegekassen), die Kassenärztliche Bundesvereinigung und die DKG) bis zuletzt nicht einigen konnten, entschied das erweiterte Bundesschiedsamt am 17.10.2016, wobei im Wesentlichen die Kritikpunkte der DKG (z.B. Finanzierung der anfallenden Kosten für das Krankenhaus für das Bereitstellen des notwendigen Fachpersonals und der Leistungserweiterung für das Überleitungsmanagement; LANR für Krankenhausärzte; Entlassmanagement für Patienten, die Bedarf haben, etc.) kein Gehör fanden.

\section{Was passiert jetzt?}

Das SGB V regelt ganz klar, dass die Klage gegen die Entscheidung des Schiedsamtes keine aufschiebende Wirkung hat, d.h. der Vertrag zunächst wirksam ist und Geltung entfaltet, obwohl Klage eingereicht wurde. Die DKG schreibt allerdings in ihrer Pressemitteilung, die Klage würde die geplante Umsetzung im Juli 2017 bis zum Abschluss des Rechtsstreits aufschieben. Unberührt davon bliebe aber der Rechtsanspruch des Patienten auf ein ordnungsgemäßes Entlassmanagement erhalten, das die Krankenhäuser weiter in bewährten Verfahren fortführen würden. Voraussichtlich wird eine vollständige Umsetzung des Vertrages zum 01.07.2017 rein faktisch kaum möglich sein. Es bleibt abzuwarten, ob es zwischen den Vertragspartnern eine Art „agreement" geben wird, was geduldet wird und was nicht. Grundsätzlich sind die getroffenen Regelungen für alle Vertragspartner verbindlich.

\section{Was regelt der Rahmenver- trag?}

Der Rahmenvertrag sieht eine Vielzahl an Regelungen für die praktische Umsetzung vor, die teilweise die Regelungen des G-BA ergänzen. So finden sich dort u.a. folgende, von Krankenhäusern zu beachtende Vorgaben:

\section{Standardisiertes Entlassmanage- ment}

Das Krankenhaus muss ein standardisiertes Entlassmanagement in multidisziplinärer Zusammenarbeit sicherstellen und schriftliche, für alle Beteiligten transparente Standards (z.B. für die Pflege: Expertenstandard Entlassmanagement in der Pflege) etablieren.

\section{Patientenindividueller Bedarf/ Aufstellung Entlassplan}

Zur Gewährleistung eines nahtlosen Übergangs der Patienten in die nachfolgenden Versorgungsbereiche muss unter Verantwortung des Krankenhausarztes durch die Anwendung eines geeigneten Assessments der patientenindividuelle Bedarf für die Anschlussversorgung möglichst frühzeitig erfasst und ein Entlassplan aufgestellt werden. Der Entlassplan umfasst dabei den voraussichtlichen Versorgungsbedarf im Anschluss an die Krankenhausbehandlung. Das Krankenhaus leitet die im Entlassplan festgelegten, voraussichtlich erforderlichen Maßnahmen frühestmöglich ein. Der Ent- 
lassplan muss für alle Mitarbeiter des Krankenhauses, die am Entlassmanagement des jeweiligen Patienten beteiligt sind, in der Patientenakte verfügbar sein. Für Patienten mit komplexem Versorgungsbedarf gelten besondere Regelungen.

\section{Entlassbrief/Nennung zuständiger Ansprechpartner}

Zusätzlich zum Entlassbrief wurde im Rahmenvertrag verpflichtend geregelt, eine Rufnummer eines zuständigen Ansprechpartners für Rückfragen der weiterbehandelnden Leistungserbringer anzugeben. Unter dieser Rufnummer muss zumindest Montag bis Freitag in der Zeit von 09:0019:00 Uhr, Samstag von 10:00-14:00 Uhr und Sonntag von 10:00-14:00 Uhr ein für das Entlassmanagement des Krankenhauses zuständiger Ansprechpartner für Rückfragen zur Verfügung stehen.

\section{Verordnungsausstellung und Hinweispflicht}

Besteht die Notwendigkeit, Arzneimittel etc. zu verordnen oder Arbeitsunfähigkeit festzustellen, erhält der Patient spätestens am Tag der Entlassung die entsprechende Verordnung bzw. Bescheinigung der Arbeitsunfähigkeit. Der Patient ist über das Erfordernis der rechtzeitigen Inanspruchnahme der verordneten Leistungen zu informieren.

\section{Regelungen zum Verord- nungsrecht}

Das Verordnungsrecht (!) ist auf die Erfordernisse des Entlassmanagements eingeschränkt (Stichwort: unmittelbare Erforderlichkeit). Die Verwendung besonders zu kennzeichnender Verordnungsblätter und konkrete Vorgaben für die Verordnungen sind im Rahmenvertrag geregelt.

Werden entsprechende Verordnungen getätigt, gelten die Bestimmungen über die vertragsärztliche Versorgung - mit allen Konsequenzen. Damit unterfallen die Krankenhäuser in diesen Bereichen sowohl den leistungsrechtlichen Vorgaben als auch den Wirtschaftlichkeitsbestimmungen aus dem GKV-Bereich - letztlich wie „normale“ Vertragsärzte. Dies ist insoweit von erheblicher Bedeutung, da Regresse im Falle von Fehlern bei der Verordnung von EntlassRezepten auch in Höhe der ambulanten
Preise (abzgl. Rabatte und Zuzahlungen) regressiert werden können, die weit über den günstigen Krankenhauspreisen liegen. Denkbar sind z.B. Prüfungen, ob die getätigte Verordnung wirklich unmittelbar erforderlich war, sich das Krankenhaus an die vorgeschriebenen Packungsgrößen gehalten hat oder die Rezepte formal korrekt ausstellt wurden.

Bei der Verordnung von Arzneimitteln sind aber noch weitere Vorgaben zu beachten:

- §115c SGB V (Arzneimitteltherapie nach Krankenhausbehandlung), dort ist unter anderem geregelt, dass das Krankenhaus nur Wirkstoffe verordnen soll, die im ambulanten Bereich auch weiterverordnet werden können.

- Verträge nach § 130a Abs. 8 SGB V (Rabattverträge zwischen Pharmazeutischen Unternehmen und einzelnen Krankenkassen)

- 130b SGB V (Vereinbarungen über Erstattungsbeträge für Arzneimittel mit Nutzenbewertung)

- 130c SGB V („freiwillige“ Vereinbarungen über Erstattungsbeträge“ zwischen Pharmazeutischen Unternehmen und Krankenkassen“)

- die Regelungen zu den Versorgungsund Wirtschaftlichkeitszielen nach $\S 84$ SGB V entsprechend

Letztlich müssen sich die verordnenden Krankenhausärzte (und ggf. das zuarbeitende Pflegepersonal) mit allen im niedergelassenen Bereich für GKV-Verordnungen von Arzneimitteln geltenden Regelungen vertraut machen (und das sind zahlreiche!). Die DKG vertritt die Auffassung, dass eine Wirtschaftlichkeitsprüfung nach vertragsärztlichen Grundsätzen gegen ein Krankenhaus nicht rechtskonform wäre, da im Widerspruchsverfahren keine Vertreter des Krankenhauses vorgesehen seien.

Grundsätzlich besteht die Möglichkeit für Krankenhäuser und Krankenkassen, eigene Vereinbarungen zu Wirtschaftlichkeitsprüfungen zu treffen. Hierdurch könnten Krankenhäuser unter Umständen das für sie völlig unkalkulierbare Risiko von Wirtschaftlichkeitsprüfungen etwas eindämmen, indem individuelle Vereinbarungen getroffen werden. Inwieweit von Seiten der Krankenkassen hierzu Interesse besteht, hängt voraussichtlich von der Größe des Krankenhauses, der Größe der Kasse und dem Verordnungsumfang ab.

\section{DKG informiert Kranken-}

\section{häuser}

Laut Rahmenvertrag informiert die DKG in geeigneter Weise über die bei der Verordnung zu beachtenden Bestimmungen; dies gilt insbesondere für die Arznei- und Betäubungsmittelverschreibungsverordnungen. Da sich die rechtlichen Rahmenbedingungen für die Verordnung von GKV-Arzneimitteln ständig ändern und z.T. regional erheblich unterscheiden, wird es spannend, wie die DKG diesem Auftrag gerecht werden soll.

\section{Freie Apothekenwahl und Verbot von Absprachen}

Bei allen verordneten Leistungen ist das Recht des Patienten auf freie Wahl des Leistungserbringers sowie $\S 128$ SGB V zu beachten, es sei denn, anderweitige gesetzliche Regelungen stehen dem entgegen. Der Patient ist ausdrücklich auf das Recht der freien Wahl des Leistungserbringers hinzuweisen. Eine Bevorzugung eines Anbieters ist nicht statthaft. Vereinbarungen oder Absprachen zwischen Krankenhäusern und Apotheken oder sonstigen Leistungserbringern veranlasster Leistungen, die auf eine Zuweisung von Patienten abzielen, sind unzulässig.

\section{LANR für Krankenhausärzte}

Die Kassenärztliche Vereinigung vergibt eine Arztnummer an Krankenhausärzte, die Leistungen im Entlassmanagement erbringen, die bei Verordnungen anzugeben ist. Die DKG spricht hier von einer Zwangsregistrierung von 50000 Krankenhausärzten. Dies würde der Intention des Gesetzgebers widersprechen, da sich der Anspruch des Patienten auf Entlassmanagement ans Krankenhaus als Institution selbst richte und nicht an den einzelnen Arzt.

Andererseits muss der Aussteller eines Rezeptes bereits aus haftungsrechtlichen Gründen ohne Frage identifizierbar sein.

\section{Entlassmanagement für alle Patienten}

Entgegen dem Wunsch der DKG, gilt das Entlassmanagement grundsätzlich für alle 
Patienten aus voll- und teilstationären Behandlungen im Krankenhaus. Letztlich besteht dieser Anspruch unabhängig davon, ob die Patienten ein Entlassmanagement benötigen oder nicht. Sollte jeder der ca. 19 Mio. Patienten pro Jahr einem Entlassmanagement unterzogen werden, bedeute das für die Kliniken zusammen einen Aufwand von rund 100.000 Arbeitstagen im Jahr, so die Berechnungen der DKG. Zu berücksichtigen ist dabei jedoch, dass der Patient freiwillig teilnimmt. Allerdings ist alleine die Dokumentation der Einwilligung bzw. Ablehnung mit nicht unerheblichem Aufwand verbunden.

\section{Keine Finanzierung für zusätzlichen Aufwand}

Auch die Forderung der DKG, die durch den erheblichen Mehraufwand anfallenden Kosten entsprechend finanziert zu bekommen, wurde vom Bundesschiedsamt abgewiesen.

\section{FAZIT}

Der im Rahmenvertrag festgelegte Aufwand für die Krankenhäuser sowohl in finanzieller Art als auch inhaltlich (Schulungen, Überwachungen von Verordnungen, Implikation von Software für E-Rezepte, Erreichbarkeit von Ärzten etc.) wird beträchtlich und teilweise in der Praxis kaum umsetzbar sein. Durch die Klage der DKG gegen den Schiedsspruch kommt nach wie vor keine Ruhe in das Thema. Die nächsten Monate werden zeigen, ob und in welcher Form noch Kompromisse zwischen den Vertragspartnern gefunden werden können. Die Frage, wie Krankenhäuser die Umsetzung aller Verpflichtungen zum Entlassmanagement ohne finanzielle Unterstützung leisten sollen, muss erlaubt sein. Die Regelungen gehen in großen Teilen vollkommen an der Realität, was ein Krankenhaus leisten kann, vorbei.

\section{Literatur}

[1] Häser I. Neuregelungen zum Entlassmanagement der Krankenhäuser. Ausweitung der Regelungen durch das Versorgungsstärkungsgesetz. klinikarzt 2015; 44: 584-585

[2] Häser I. Entlassmedikation - G-BA fasst ausführlichen Beschluss. klinikarzt 2016; 45: $274-275$

Autor

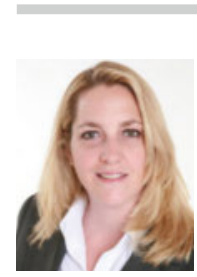
Dr. iur. Isabel Häser Rechtsanwältin Fachanwältin für Medizinrecht

Haimhauser Str. 1

80802 München haeser@kanzlei-haeser.de 